\title{
SUBEXPONENTIAL TAIL ASYMPTOTICS FOR A RANDOM WALK WITH RANDOMLY PLACED ONE-WAY NODES
}

\author{
Nina GANTERT \\ Institut fur Mathematische Stochastik, Universitàt Karlsruhe, Englerstr. 2, 76128 Karlsruhe, Germany
}

Received 3 December 1998, revised 7 May 2001

ABSTRACT. - Let $p \in] 0,1 / 2]$ and assign to the integers random variables $\left\{\omega_{x}\right\}$, taking only the two values 1 and $p$, which serve as an environment. This environment defines a random walk $\left\{X_{n}\right\}$ which, when at $x$, moves one step to the right with probability $\omega_{x}$, and one step to the left with probability $1-\omega_{x}$. In particular, at the nodes, i.e., at the locations $x$ with $\omega_{x}=1$, no backtrack is possible. We will assume that the speed $v_{\alpha}$ of the random walk is positive. We then investigate, for $v<v_{\alpha}$, the decay of the probabilities $\mathbb{P}_{\omega}\left[X_{n} / n \leqslant v\right]$ (for fixed environment) and $\mathbb{P}\left[X_{n} / n \leqslant v\right]$ (averaged over the environment). These probabilities decay subexponentially and there is a wide range of possible normalizations, depending on the distribution of the lengths of the intervals without nodes. We show that in fact only the behaviour of the length of the largest interval without nodes (contained in $[0, n]$ ) matters. () 2002 Éditions scientifiques et médicales Elsevier SAS

AMS classification: 60J15; 60F10; 82C44; 60J80

Keywords: Random walk in random environment; Large deviations; Extreme values

RÉSUMÉ. - Soit $p \in] 0,1 / 2]$ et soit $\left(\omega_{x}\right)_{x \in \mathbb{Z}}$ une suite de variables prenant les deux valeurs 1 et $p$, utilisée comme environnement. Dans cet environnement, on définit une marche aléatoire, qui va de $x$ à $x+1$ avec probabilité $\omega_{x}$, et de $x$ à $x-1$ avec probabilité $1-\omega_{x}$. Quand $x$ est un noeud (un point où $\omega_{x}=1$ ), la marche après avoir dépassé $x$ ne peut plus jamais revenir en $x-1$. Nous faisons l'hypothèse que cette marche a une vitesse strictement positive, que nous notons $v_{a}$. Nous étudions, pour $v<v_{a}$, la décroissance des probabilités $\mathbb{P}_{\omega}\left[X_{n} / n \leqslant v\right]$ (pour un environnement fixé) et $\mathbb{P}\left[X_{n} / n \leqslant v\right]$ (moyennée sur les environnements). Ces probabilités décroissent sousexponentiellement et la normalisation dépend de la loi des longueurs des intervalles sans noeuds. Plus précisément, nous montrons que c'est seulement l'intervalle sans noeuds le plus long qui compte. ( 2002 Éditions scientifiques et médicales Elsevier SAS

Mots Clés: Marche aléatoire dans un environnement aléatoire; Grandes déviations; Valeurs extrêmes 


\section{Introduction and statement of results}

We will consider the following particular case of a one-dimensional random walk in random environment (RWRE). We first choose the environment, placing one-way nodes at certain random locations. The random walk in this environment is a nearest-neighbour random walk which is forced to go right at the nodes and has fixed (deterministic) probabilities to jump right or left on the intervals without nodes. Let $\left.p \in] 0, \frac{1}{2}\right]$ and $\Omega:=\{1, p\}^{\mathbb{Z}}$. We denote the elements of $\Omega$ by $\omega=\left(\omega_{x}\right)_{x \in \mathbb{Z}}$. The sequence $\left(\omega_{x}\right)_{x \in \mathbb{Z}}$ will serve as an environment. We assume that the distribution $\alpha$ of $\left(\omega_{x}\right)_{x \in \mathbb{Z}}$ is stationary and ergodic with respect to the shift transformation. Let $\bar{\alpha}$ be the conditional distribution of $\alpha$, given that $\omega_{0}=1$, i.e., $\bar{\alpha}:=\alpha\left(\cdot \mid \omega_{0}=1\right)$. Let $\ell_{1}(\omega), \ell_{2}(\omega), \ldots$ be the lengths of the successive intervals without nodes, i.e.,

$$
\begin{gathered}
\ell_{1}(\omega):=\inf \left\{i \geqslant 1: \omega_{i}=1\right\}, \\
\ell_{1}(\omega):=\inf \left\{i \geqslant 1: \omega_{\ell_{1}+\cdots+\ell_{k-1}+i}=1\right\}, \quad k \geqslant 2 .
\end{gathered}
$$

Then the random variables $\ell_{1}, \ell_{2}, \ldots$ form a stationary, ergodic sequence under $\bar{\alpha}$. For every fixed $\omega$ let $X=\left(X_{n}\right)_{n \geqslant 0}$ be the Markov chain on $\mathbb{Z}$ starting at $X_{0}=0$, with transition probabilities

$$
\mathbf{P}_{\omega}\left(X_{n+1}=y \mid X_{n}=x\right)= \begin{cases}\omega_{x} & \text { if } y=x+1, \\ 1-\omega_{x} & \text { if } y=x-1, \\ 0 & \text { otherwise. }\end{cases}
$$

We will denote the distribution of $\left(X_{n}\right)$, given $\omega$ (or, equivalently, given $\left.\ell_{1}, \ell_{2}, \ldots\right)$, with $\mathbf{P}_{\omega}$, and the joint distribution of $\left(\omega,\left(X_{n}\right)\right)$ under $\bar{\alpha} \times \mathbf{P}_{\omega}$ with $\mathbf{P}$. The process $\left(X_{n}\right)$ was introduced in [13], in the case where $\alpha$ is a product measure, and studied further in [2], in the case where $\ell_{1}, \ell_{2}, \ldots$ are i.i.d. random variables. Limit laws for the distribution of $X_{n}$, suitably normalized, were investigated in [13] and in [2]. Let

$$
\tau_{\ell_{1}}:=\inf \left\{j: X_{j}=\ell_{1}\right\}
$$

and

$$
\tau_{\ell_{k+1}}:=\inf \left\{j>\tau_{\ell_{1}}+\cdots+\tau_{\ell_{k}}: X_{j}=\ell_{1}+\cdots+\ell_{k+1}\right\}-\left(\tau_{\ell_{1}}+\cdots+\tau_{\ell_{k}}\right), \quad k \geqslant 2 .
$$

Under appropriate assumptions, the random walk is moving to the right with a positive speed, see [1] and [2].

LEMMA 1. - With

$$
v_{\alpha}:=\frac{\mathbf{E}\left[\ell_{1}\right]}{\mathbf{E}\left[\tau_{\ell_{1}}\right]},
$$

where $v_{\alpha}=0$ if $\mathbf{E}\left[\tau_{\ell_{1}}\right]=\infty$, we have

$$
\frac{X_{n}}{n} \underset{n \rightarrow \infty}{\longrightarrow} v_{\alpha} \quad \mathbf{P} \text {-a.s. }
$$


i.e., $X_{n} / n \rightarrow v_{\alpha} \mathbf{P}_{\omega}$-a.s., for $\bar{\alpha}$-a.a. $\omega$.

Let

$$
\rho:=\frac{1-p}{p} \geqslant 1
$$

Later, see (45) and (56), we will give an explicit formula for $\mathbf{E}\left[\tau_{\ell_{1}}\right]$, leading to

$$
\frac{1}{v_{\alpha}}=-\frac{\rho+1}{\rho-1}+\frac{1}{\mathbf{E}\left[\ell_{1}\right]}\left(\mathbf{E}\left[\rho^{\ell_{1}}\right]-1\right) \frac{2 \rho}{(\rho-1)^{2}} \quad \text { if } p<\frac{1}{2}
$$

and

$$
v_{\alpha}=\frac{\mathbf{E}\left[\ell_{1}\right]}{\mathbf{E}\left[\ell_{1}^{2}\right]} \quad \text { if } p=\frac{1}{2} .
$$

In particular, if $p<\frac{1}{2}, v_{\alpha}>0$ if and only if $\mathbf{E}\left[\rho^{\ell_{1}}\right]<\infty$ and, if $p=\frac{1}{2}, v_{\alpha}>0$ if and only if $\mathbf{E}\left[\ell_{1}^{2}\right]<\infty$. We will always assume that $v_{\alpha}>0$. Due to (7), for each $v<v_{\alpha}$, the probabilities $\mathbf{P}_{\omega}\left[X_{n} / n \leqslant v\right]$ and $\mathbf{P}\left[X_{n} / n \leqslant v\right]$ go to 0 . The goal of this paper is to investigate the rate of this decay. For $v=0$, one can show that $\mathbf{P}_{\omega}\left[X_{n} / n \leqslant 0\right] \leqslant$ $\mathbf{P}_{\omega}\left[\tau_{\ell_{1}}>n\right]$ decays exponentially in $n$, for $v<0$, we trivially have $\mathbf{P}_{\omega}\left[X_{n} / n \leqslant v\right]=0$. Throughout the paper, we will only consider strictly positive values of $v$. In the general RWRE model, $\left(\omega_{x}\right)$ is an ergodic sequence of random variables with values in $[0,1]$. Large deviations for this model were investigated in [8,5,3], see also the survey paper [7]. If $\omega_{x}>\frac{1}{2}$, for all $x$, it is known that $v_{\alpha}>0$ and that for $v<v_{\alpha}, \mathbf{P}_{\omega}\left[X_{n} / n \leqslant v\right]$ and $\mathbf{P}\left[X_{n} / n \leqslant v\right]$ decay exponentially in $n$. The exponential rate of decay was identified in [8] and [3]. In contrast, we treat here an environment with "mixed drifts and positive speed". It was shown in [3] that for such environments, under mild assumptions on $\alpha$, $\mathbf{P}_{\omega}\left[X_{n} / n \leqslant v\right]$ and $\mathbf{P}\left[X_{n} / n \leqslant v\right]$ decay subexponentially in $n$ for $0<v<v_{\alpha}$. For the i.i.d. environment case, i.e., if $\alpha$ is a product measure, subexponential asymptotics were derived in [5] and [12] for the decay of $\mathbf{P}\left[X_{n} / n \leqslant v\right]$, and in [6] and [11], for the decay of $\mathbf{P}_{\omega}\left[X_{n} / n \leqslant v\right]$. Strictly speaking, not all the above mentioned results apply in our situation, since some of them assume that the support of the distribution of $\omega_{0}$ is contained in a compact subset of ]0,1[. In this paper, we prove subexponential asymptotics for the random walk with randomly placed one-way nodes. We show that under appropriate conditions (see Theorem 1), $\log \mathbf{P}_{\omega}\left[X_{n} / n \leqslant v\right]$ has "random fluctuations" and there is no deterministic normalization. The same behaviour is conjectured for the general RWRE model, see [7]), and was proved for a random walk with one-way nodes in an i.i.d. environment (see Theorem 9 in [7]). Let

$$
N_{\ell}(n):=\max \left\{j: \ell_{1}+\cdots+\ell_{j} \leqslant n\right\}, \quad \ell_{\max }(n):=\max _{1 \leqslant j \leqslant N_{\ell}(n)} \ell_{j}(\omega),
$$

and

$$
N(n):=\min \left\{j: \ell_{1}+\cdots+\ell_{j} \geqslant n\right\}, \quad \ell_{\mathrm{MAX}}(n):=\max _{1 \leqslant j \leqslant N(n)} \ell_{j}(\omega) .
$$

Let

$$
T_{n}:=\inf \left\{j: X_{j}=n\right\}
$$


Throughout the paper, $a_{n}, b_{n}, c_{n}$ and $d_{n}$ are positive, increasing functions of $n$ with $a_{n} \rightarrow \infty, b_{n} \rightarrow \infty, c_{n} \rightarrow \infty, d_{n} \rightarrow \infty$ and $b_{n} / a_{n} \rightarrow 0, c_{n} / a_{n} \rightarrow 0$ and $d_{n} / a_{n} \rightarrow 0$. We will further assume that $a_{n}$ is slowly varying, i.e. that for each $k \geqslant 1, a_{n k} / a_{n} \rightarrow 1$ for $n \rightarrow \infty$. Our main results are the following.

THEOREM 1. - Assume that $p<\frac{1}{2}$ and $\mathbf{E}\left[\rho^{\ell_{1}}\right]<\infty$. Assume further that $a_{n}, b_{n}$ and $c_{n}$ as above exist such that

$$
\mathbf{P}\left[\ell_{\max }(n) \geqslant a_{n}+b_{n} \text { for infinitely many } n\right]=1
$$

and

$$
\mathbf{P}\left[\ell_{\max }(n) \leqslant a_{n}-c_{n} \text { for infinitely many } n\right]=1 .
$$

Then we have, for each $u>1 / v_{\alpha}$,

$$
\begin{gathered}
\liminf _{n \rightarrow \infty} \frac{\rho^{a_{n}}}{n} \log \mathbf{P}_{\omega}\left[T_{n} \geqslant n u\right]=-\infty \quad \text { P-a.s. } \\
\limsup _{n \rightarrow \infty} \frac{\rho^{a_{n}}}{n} \log \mathbf{P}_{\omega}\left[T_{n} \geqslant n u\right]=0 \quad \text { P-a.s. }
\end{gathered}
$$

Assume in addition that for all $k \in \mathbb{N}$,

$$
\limsup _{n \rightarrow \infty}\left(a_{k n}-a_{n}\right)<\infty .
$$

Then, for $0<v<v_{\alpha}$,

$$
\begin{aligned}
& \liminf _{n \rightarrow \infty} \frac{\rho^{a_{n}}}{n} \log \mathbf{P}_{\omega}\left[X_{n} \leqslant n v\right]=-\infty \quad \mathbf{P} \text {-a.s. }, \\
& \limsup _{n \rightarrow \infty} \frac{\rho^{a_{n}}}{n} \log \mathbf{P}_{\omega}\left[X_{n} \leqslant n v\right]=0 \quad \mathbf{P} \text {-a.s. }
\end{aligned}
$$

Remarks. - 1. In particular, (19) and (20) show that there is no deterministic function $f$ such that,

$$
-\infty<\liminf _{n \rightarrow \infty} \frac{1}{f(n)} \log \mathbf{P}_{\omega}\left[X_{n} \leqslant n v\right] \leqslant \limsup _{n \rightarrow \infty} \frac{1}{f(n)} \log \mathbf{P}_{\omega}\left[X_{n} \leqslant n v\right]<0 \quad \text { P-a.s. }
$$

2. We conjecture that (18) is always satisfied under the previous assumptions of Theorem 1 (note that they imply (36) below!), but we were not able to show this.

THEOREM 2. - Assume that $p=\frac{1}{2}$ and $\mathbf{E}\left[\ell_{1}^{\gamma}\right]<\infty$ for some $\gamma>2$. Assume that for $a_{n}$ and $d_{n}$,

$$
\lim _{n \rightarrow \infty} \frac{\ell_{\max }(n)}{a_{n}}=1 \quad \text { P-a.s. }
$$

and

$$
\mathbf{P}\left[\ell_{\max }(n) \geqslant a_{n}+d_{n} \text { for infinitely many } n\right]=0 .
$$


Then we have, for each $u>1 / v_{\alpha}$,

$$
\lim _{n \rightarrow \infty} \frac{a_{n}^{2}}{n} \log \mathbf{P}_{\omega}\left[T_{n} \geqslant n u\right]=-\frac{\pi^{2}}{8}\left(u-\frac{1}{v_{\alpha}}\right) \quad \mathbf{P} \text {-a.s. }
$$

and, for $0<v<v_{\alpha}$,

$$
\lim _{n \rightarrow \infty} \frac{a_{n}^{2}}{n} \log \mathbf{P}_{\omega}\left[X_{n} \leqslant n v\right]=-\frac{\pi^{2}}{8}\left(1-\frac{v}{v_{\alpha}}\right) \quad \mathbf{P} \text {-a.s. }
$$

The following theorem gives statements for the decay of $\mathbf{P}\left[T_{n} \geqslant n u\right]$ and $\mathbf{P}\left[X_{n} \leqslant n v\right]$. It is a general fact that the decay of these averaged probabilities is either slower or the same than for fixed environment.

THEOREM 3. - Assume that $\ell_{1}, \ell_{2}, \ldots$ are i.i.d., $p<1 / 2$ and $\mathbf{E}\left[\rho^{3 \ell_{1}}\right]<\infty$. Assume $\varphi(n)$ is an increasing, slowly varying function such that $\varphi(n) \rightarrow \infty$ and for some continuous function $\kappa(\cdot)>0$,

$$
\lim _{n \rightarrow \infty} \frac{1}{\varphi(n)} \log \mathbf{P}\left[\ell_{\max }(n) \geqslant t \log n\right]=-\kappa(t) \quad \forall t .
$$

Then we have, for each $u>1 / v_{\alpha}$,

$$
\lim _{n \rightarrow \infty} \frac{1}{\varphi(n)} \log \mathbf{P}\left[T_{n} \geqslant n u\right]=-\kappa\left(\frac{1}{\log \rho}\right),
$$

and, for $0<v<v_{\alpha}$,

$$
\lim _{n \rightarrow \infty} \frac{1}{\varphi(n)} \log \mathbf{P}\left[X_{n} \leqslant n v\right]=-\kappa\left(\frac{1}{\log \rho}\right) .
$$

Remark. - Our proof of Theorem 3 requires that $\ell_{1}, \ell_{2}, \ldots$ are i.i.d. We don't know if this assumption is necessary for (27) and (28) to hold.

The main ingredients of our proofs are exit time asymptotics for the intervals without nodes, combined with the ergodic theorem applied to the environment sequence.

\section{Proofs}

Sketch of the proof of Lemma 1. - It is well-known that

$$
\lim _{n \rightarrow \infty} \frac{X_{n}}{n}=\left(\lim _{n \rightarrow \infty} \frac{T_{n}}{n}\right)^{-1} \quad \text { P-a.s. }
$$

where both sides equal 0 if $T_{n} / n \rightarrow \infty$, see [13]. We will show that

$$
\frac{T_{n}}{n} \underset{n \rightarrow \infty}{\longrightarrow} \frac{\mathbf{E}\left[\tau_{\ell_{1}}\right]}{\mathbf{E}\left[\ell_{1}\right]} \quad \mathbf{P} \text {-a.s. }
$$


First note that due to the ergodic theorem,

$$
\frac{N_{\ell}(n)}{n} \underset{n \rightarrow \infty}{\longrightarrow} \frac{1}{\mathbf{E}\left[\ell_{1}\right]} \text { and } \quad \frac{N(n)}{n} \underset{n \rightarrow \infty}{\longrightarrow} \frac{1}{\mathbf{E}\left[\ell_{1}\right]} \quad \text { P-a.s. }
$$

Further, the ergodic theorem implies that

$$
\frac{1}{n} \sum_{j=1}^{n} \tau_{\ell_{j}} \underset{n \rightarrow \infty}{\longrightarrow} \mathbf{E}\left[\tau_{\ell_{1}}\right] \quad \text { P-a.s. }
$$

But

$$
\sum_{j=1}^{N_{\ell}(n)} \tau_{\ell_{j}} \leqslant T_{n} \leqslant \sum_{j=1}^{N(n)} \tau_{\ell_{j}}
$$

hence

$$
\frac{N_{\ell}(n)}{n} \frac{1}{N_{\ell}(n)} \sum_{j=1}^{N_{\ell}(n)} \tau_{\ell_{j}} \leqslant \frac{T_{n}}{n} \leqslant \frac{N(n)}{n} \frac{1}{N(n)} \sum_{j=1}^{N(n)} \tau_{\ell_{j}},
$$

and (30) follows.

We note for further reference that $\mathbf{E}\left[\ell_{1}\right]<\infty$ implies

$$
\frac{\ell_{\mathrm{MAX}}(n)}{n} \underset{n \rightarrow \infty}{\longrightarrow} 0 \text { P-a.s. }
$$

To show (34), note that $\ell_{\operatorname{MAX}}(n) \leqslant \max _{1 \leqslant j \leqslant n} \ell_{j}$, and $\ell_{1}, \ell_{2}, \ldots$ are positive random variables with the same distribution and $\mathbf{E}\left[\ell_{1}\right]<\infty$. Let $c>0$. Then,

$$
\sum_{j=1}^{\infty} \mathbf{P}\left[\ell_{j} \geqslant c j\right]=\sum_{j=1}^{\infty} \mathbf{P}\left[\ell_{1} \geqslant c j\right]=\mathbf{E}\left[\frac{\ell_{1}}{c}\right]<\infty
$$

and the Borel-Cantelli lemma implies that $\mathbf{P}\left[\ell_{j} \geqslant c j\right.$ for infinitely many $\left.j\right]=0$. We conclude that also $\mathbf{P}\left[\max _{1 \leqslant j \leqslant n} \ell_{j} \geqslant c n\right.$ for infinitely many $\left.n\right]=0$. We also note that under the hypothesis of Theorem 1, we have

$$
\limsup _{n \rightarrow \infty} \frac{a_{n}}{\log n}<\infty
$$

To prove (36), taking into account (14), it is enough to show that, for some $c>0$,

$$
\mathbf{P}\left[\ell_{\max }(n) \geqslant c \log n \text { for infinitely many } n\right]=0 .
$$

But

$$
\mathbf{P}\left[\ell_{\max }(n) \geqslant c \log n\right] \leqslant n \mathbf{P}\left[\ell_{1} \geqslant c \log n\right] \leqslant n \mathbf{E}\left[\rho^{\ell_{1}}\right] \rho^{-c \log n}=n^{1-c \log \rho} \mathbf{E}\left[\rho^{\ell_{1}}\right]
$$

and we see that, for $c>2 / \log \rho$, (37) follows with the Borel-Cantelli lemma. 
Proof of Theorem 1. - The proof of (17) is the same as the proof of Theorem 9 in [7]. We will first give an estimate for the exit time of an interval without node. Let $P_{x}$ be the distribution of the random walk $\left(X_{j}\right)$, started at $x \in\{0,1, \ldots, \ell\}$, with $\omega_{0}=1$, $\omega_{1}=\omega_{2}=\cdots=\omega_{\ell-1}=p$, let $\bar{\tau}_{\ell}:=\inf \left\{j: X_{j}=\ell\right\}$ and $\bar{\tau}_{0}:=\inf \left\{j: X_{j}=0\right\}$. Then, with $\rho$ defined in (8),

$$
P_{1}\left(\bar{\tau}_{0}<\bar{\tau}_{\ell}\right)=\frac{\rho^{\ell}-\rho}{\rho^{\ell}-1} \geqslant \frac{\rho^{\ell-1}-1}{\rho^{\ell-1}} \quad \text { and } \quad P_{1}\left(\bar{\tau}_{\ell} \geqslant n\right) \geqslant P_{1}\left(\bar{\tau}_{0}<\bar{\tau}_{\ell}\right)^{n} \geqslant\left(1-\frac{1}{\rho^{\ell-1}}\right)^{n} \text {. }
$$

We give a lower bound on $\mathbf{P}_{\omega}\left[T_{n} \geqslant n u\right]$ by simply picking the largest interval without nodes.

$$
\mathbf{P}_{\omega}\left[T_{n} \geqslant n u\right] \geqslant P_{1}\left[\bar{\tau}_{\ell_{\max }(n)} \geqslant[n u]\right] \geqslant\left(1-\frac{1}{\rho^{\ell_{\max }(n)-1}}\right)^{[n u]} \geqslant\left(1-\frac{1}{\rho^{\ell_{\max }(n)-1}}\right)^{n u}
$$

where $[n u]$ denotes the integer part of $n u$. We choose a (random) subsequence $\left(n_{k}\right)$ such that $n_{k} \rightarrow \infty$ for $k \rightarrow \infty$ and

$$
\ell_{\max }\left(n_{k}\right) \geqslant a_{n_{k}}+b_{n_{k}}
$$

for all $k$. Then, due to (40), for each $\varepsilon>0$,

$$
\begin{aligned}
\log \mathbf{P}_{\omega}\left[T_{n_{k}} \geqslant n_{k} u\right] & \geqslant \log \left(1-\frac{1}{\rho^{\ell_{\max }\left(n_{k}\right)-1}}\right)^{n_{k} u} \\
& \geqslant-n_{k} u \rho^{-\left(a_{n_{k}}+b_{n_{k}}\right)}(1+\varepsilon) \quad \text { P-a.s. }
\end{aligned}
$$

for $k$ large enough. Hence

$$
\lim _{k \rightarrow \infty} \frac{\rho^{a_{n_{k}}}}{n_{k}} \log \mathbf{P}_{\omega}\left[T_{n_{k}} \geqslant n_{k} u\right]=0 \quad \mathbf{P} \text {-a.s. }
$$

and (17) follows. To show (16), we first prove the following formula for the exit time of a random walk with reflection at 0 .

LeMmA 2. - Let $\omega_{0}=1, \omega_{1}=\omega_{2}=\cdots=\omega_{\ell-1}=p<\frac{1}{2}, X_{0}=0, \bar{\tau}_{\ell}:=\inf \left\{j: X_{j}=\right.$ $\ell\}$ and $g(\ell):=g(\lambda, \ell)=E_{0}\left[\exp \left(\lambda \bar{\tau}_{\ell}\right)\right]$. Let

$$
\begin{aligned}
& \eta_{1}=\eta_{1}(\lambda)=\frac{1}{2 p}\left(\mathrm{e}^{-\lambda}+\sqrt{\mathrm{e}^{-2 \lambda}-4 p q}\right), \\
& \eta_{2}=\eta_{2}(\lambda)=\frac{1}{2 p}\left(\mathrm{e}^{-\lambda}-\sqrt{\mathrm{e}^{-2 \lambda}-4 p q}\right)
\end{aligned}
$$

with $q:=1-p$, and

$$
\lambda_{\text {crit }}:=\lambda_{\text {crit }}(\ell)=\sup \left\{\lambda>0: \mathrm{e}^{-2 \lambda}>4 p q, \eta_{2}^{\ell}\left(\mathrm{e}^{\lambda} \eta_{1}-1\right)>\eta_{1}^{\ell}\left(\mathrm{e}^{\lambda} \eta_{2}-1\right)\right\} .
$$


Then, for $0 \leqslant \lambda<\lambda_{\text {crit }}$, we have

$$
g(\ell)=\frac{\mathrm{e}^{\lambda}\left(\eta_{1}-\eta_{2}\right)}{\eta_{2}^{\ell}\left(\mathrm{e}^{\lambda} \eta_{1}-1\right)-\eta_{1}^{\ell}\left(\mathrm{e}^{\lambda} \eta_{2}-1\right)} .
$$

As a consequence, we have

$$
E_{0}\left[\bar{\tau}_{\ell}\right]=\left.\frac{\mathrm{d}}{\mathrm{d} \lambda} g(\ell)\right|_{\lambda=0}=-\ell \frac{\rho+1}{\rho-1}+\left(\rho^{\ell}-1\right) \frac{2 \rho}{(\rho-1)^{2}} .
$$

Proof. - See [7], proof of Lemma 6.

Remark. - We note for further reference that, using $E_{0}\left[\left(\bar{\tau}_{\ell}\right)^{m}\right]=\left.\frac{\mathrm{d}^{m}}{\mathrm{~d} \lambda^{m}} g(\ell)\right|_{\lambda=0}, m=$ $1,2, \ldots$, one can check that $\mathbf{E}\left[\left(\tau_{\ell_{1}}\right)^{2}\right]<\infty$ if and only if $\mathbf{E}\left[\rho^{2 \ell_{1}}\right]<\infty$ and

$$
\mathbf{E}\left[\left(\tau_{\ell_{1}}\right)^{3}\right]<\infty \quad \text { if and only if } \mathbf{E}\left[\rho^{3 \ell_{1}}\right]<\infty \text {. }
$$

As usual, we start the proof of the upper bound with Chebyshev's inequality. Fix $C>0$ and let $\lambda_{n}=C \rho^{-a_{n}}$. We will specify a subsequence $\left(\tilde{n}_{k}\right)$ such that

$$
\mathbf{P}_{\omega}\left[T_{\tilde{n}_{k}} \geqslant \tilde{n}_{k} u\right] \leqslant \mathbf{E}_{\omega}\left[\mathrm{e}^{\lambda \tilde{n}_{k} T_{\tilde{n}_{k}}}\right] \mathrm{e}^{-\lambda_{\tilde{n}_{k}} \tilde{n}_{k} u} \leqslant \prod_{j=1}^{N\left(\tilde{n}_{k}\right)} g\left(\ell_{j}(\omega)\right) \mathrm{e}^{-\lambda_{\tilde{n}_{k}} \tilde{n}_{k} u} \quad \mathbf{P} \text {-a.s. }
$$

We can assume w.l.o.g. that (15) still holds true if we replace $\ell_{\max }(n)$ with $\ell_{\operatorname{MAX}}(n)$. Define the (random) subsequence $\left(\tilde{n}_{k}\right)$ such that $\tilde{n}_{k} \rightarrow \infty$ and

$$
\ell_{\operatorname{MAX}}\left(\tilde{n}_{k}\right) \leqslant a_{\tilde{n}_{k}}-c_{\tilde{n}_{k}} \quad \text { P-a.s. }
$$

We have to show that, for $k$ large enough, $\lambda_{\tilde{n}_{k}}<\lambda_{\text {crit }}$ is satisfied for our choice of $\lambda_{n}$ and for all intervals along our subsequence. It suffices to show that for $\eta_{1}=\eta_{1}\left(\lambda_{\tilde{n}_{k}}\right)$ and $\eta_{2}=\eta_{2}\left(\lambda_{\tilde{n}_{k}}\right)$

$$
\left(\frac{\eta_{1}}{\eta_{2}}\right)^{\ell_{\operatorname{MAX}\left(\tilde{n}_{k}\right)}}\left(\mathrm{e}^{\lambda_{\tilde{n}_{k}}} \eta_{2}-1\right) \underset{k \rightarrow \infty}{\longrightarrow} 0 \quad \text { P-a.s. }
$$

We follow the proof of (62) in [7], and then, taking logarithms in (47), we proceed exactly as in the proof of (78) in [7], except that that there the explicit values of $\mathbf{E}\left[\ell_{1}\right]$ and $\mathbf{E}\left[\rho^{\ell_{1}}\right]$ were plugged in, and instead of (78) in [7], we end up with the statement that, P-a.s.,

$$
\begin{aligned}
\liminf _{n \rightarrow \infty} \frac{\rho^{a_{n}}}{n} \log \mathbf{P}_{\omega}\left[T_{n} \geqslant n u\right] \\
\quad \leqslant \limsup _{k \rightarrow \infty} \frac{\rho^{a_{\tilde{n}_{k}}}}{\tilde{n}_{k}} \log \mathbf{P}_{\omega}\left[T_{\tilde{n}_{k}} \geqslant \tilde{n}_{k} u\right] \\
\quad \leqslant-\frac{C}{q-p}-C \frac{1}{\mathbf{E}\left[\ell_{1}\right]} \frac{2 q p}{(q-p)^{2}}+C \frac{1}{\mathbf{E}\left[\ell_{1}\right]} \frac{2 q p}{(q-p)^{2}} \mathbf{E}\left[\rho^{\ell_{1}}\right]-C u \\
\quad=C\left(-\frac{\rho+1}{\rho-1}-\frac{1}{\mathbf{E}\left[\ell_{1}\right]} \frac{2 \rho}{(\rho-1)^{2}}+\frac{1}{\mathbf{E}\left[\ell_{1}\right]} \mathbf{E}\left[\rho^{\ell_{1}}\right] \frac{2 \rho}{(\rho-1)^{2}}\right)-C u
\end{aligned}
$$




$$
=C\left(\frac{1}{v_{\alpha}}-u\right)=-C\left(u-\frac{1}{v_{\alpha}}\right) .
$$

(16) now follows since $C>0$ was arbitrary.

The statements for $\left(X_{n}\right)$ follow from the statements for $\left(T_{n}\right)$ by renewal duality. More precisely, we have

LEMMA 3. - Under the assumptions of Theorems 1, 2 or 3, the following holds true. For $\mathbf{P}$-a.a. $\omega$, there is, for each $\varepsilon>0, n_{0}(\omega)$ such that for $n \geqslant n_{0}(\omega)$,

$$
\mathbf{P}_{\omega}\left[T_{n} \geqslant[n u]\right] \leqslant \mathbf{P}_{\omega}\left[X_{[n u]} \leqslant n\right] \leqslant \mathbf{P}_{\omega}\left[T_{[n(1+\varepsilon)]} \geqslant[n u]\right] .
$$

Proof. - The first inequality in (51) is obvious. To show the second inequality, note that

$$
\begin{aligned}
\mathbf{P}_{\omega}[ & \left.X_{[n u]} \leqslant n\right]-\mathbf{P}_{\omega}\left[T_{[n(1+\varepsilon)]} \geqslant[n u]\right] \\
& \leqslant \sum_{j=[n(1+\varepsilon)]}^{[n u]} \mathbf{P}_{\omega}\left[T_{[n(1+\varepsilon)]}=j, X_{[n u]} \leqslant n\right] \\
& \leqslant[n u] \mathbf{P}_{\omega}\left[\exists j, m \text { such that } X_{j}=[n(1+\varepsilon)], X_{j+m} \leqslant n\right] \\
& \leqslant[n u] I_{\left\{\ell_{\operatorname{MAX}}([n(1+\varepsilon)]) \geqslant[n \varepsilon]\right\}} \\
& =0 .
\end{aligned}
$$

for $n$ large enough, since $\ell_{\text {MAX }}(n) / n \rightarrow 0$ P-a.s. due to (34).

To prove (20), let $\varepsilon>0$ and $u=\varepsilon+1 / v>1 / v_{\alpha}$. We have

$$
\begin{aligned}
0 & \geqslant \limsup _{n \rightarrow \infty} \frac{\rho^{a_{n}}}{n} \log \mathbf{P}_{\omega}\left[X_{n} \leqslant n v\right] \\
& \geqslant \limsup _{n \rightarrow \infty} \frac{\rho^{a_{[n u]}}}{[n u]} \log \mathbf{P}_{\omega}\left[X_{[n u]} \leqslant[n u] v\right] \\
& \geqslant \limsup _{n \rightarrow \infty} \frac{\rho^{a_{[n u]}}}{[n u]} \log \mathbf{P}_{\omega}\left[X_{[n u]} \leqslant n\right] \\
& \geqslant \limsup _{n \rightarrow \infty} \frac{\rho^{a_{[n u]}}}{[n u]} \frac{n}{\rho^{a_{n}}} \frac{\rho^{a_{n}}}{n} \log \mathbf{P}_{\omega}\left[T_{n} \geqslant n u\right] \\
& \geqslant-\liminf _{n \rightarrow \infty} \frac{\rho^{a_{[n u]}}}{[n u]} \frac{n}{\rho^{a_{n}}} \frac{-\rho^{a_{n}}}{n} \log \mathbf{P}_{\omega}\left[T_{n} \geqslant n u\right] \\
& \geqslant \limsup _{n \rightarrow \infty}\left(\frac{\rho^{a_{[n u]}}}{[n u]} \frac{n}{\rho^{a_{n}}}\right)\left(-\liminf _{n \rightarrow \infty} \frac{-\rho^{a_{n}}}{n} \log \mathbf{P}_{\omega}\left[T_{n} \geqslant n u\right]\right) \\
& \geqslant \limsup _{n \rightarrow \infty}\left(\frac{\rho^{a_{[n u]}}}{[n u]} \frac{n}{\rho^{a_{n}}}\right) \limsup \frac{\rho^{a_{n}}}{n} \log \mathbf{P}_{\omega}\left[T_{n} \geqslant n u\right] \\
& \geqslant 0,
\end{aligned}
$$

where the last inequality follows since $\lim \sup _{n \rightarrow \infty} \frac{\rho^{a_{n}}}{n} \log \mathbf{P}_{\omega}\left[T_{n} \geqslant n u\right]=0$ due to Lemma 3 and $\lim \sup _{n \rightarrow \infty}\left(\frac{\rho^{a}[n u]}{[n u]} \frac{n}{\rho^{a_{n}}}\right)<\infty$ due to (18). The proof of (19) is similar.

Proof of Theorem 2. - We have the following analogue of Lemma 2. 
LEMMA 4. - Let $\ell \in \mathbb{N}, \omega_{0}=1, \omega_{1}=\omega_{2}=\cdots=\omega_{\ell-1}=\frac{1}{2}, \quad X_{0}=0, \bar{\tau}_{\ell}:=$ $\inf \left\{j: X_{j}=\ell\right\}$ and $\widetilde{g}(\ell):=\widetilde{g}(\ell, \lambda)=E_{0}\left[\exp \left(\lambda \bar{\tau}_{\ell}\right)\right]$. Let

$$
\widetilde{\lambda}_{\text {crit }}:=\tilde{\lambda}_{\text {crit }}(\ell)=-\log \left(\cos \frac{\pi}{2 \ell}\right) .
$$

Then, for $0 \leqslant \lambda<\widetilde{\lambda}_{\text {crit, }}$ we have

$$
\widetilde{g}(\ell)=\frac{1}{\cos \left(\ell \arccos \mathrm{e}^{-\lambda}\right)} .
$$

Proof. - Let $\omega_{i}=\frac{1}{2},-\ell+1 \leqslant i \leqslant \ell-1$ and $\widetilde{\tau}_{\ell}:=\inf \left\{j:\left|X_{j}\right|=\ell\right\}$. Then $\widetilde{\tau}_{\ell}$ has the same distribution as $\bar{\tau}_{\ell}$. Let $\widetilde{g}_{x}(\ell):=E_{x}\left[\exp \left(\lambda \widetilde{\tau}_{\ell}\right)\right]$. We have $\widetilde{g}_{\ell}(\ell)=\widetilde{g}_{-\ell}(\ell)=1$ and $\widetilde{g}_{x}(\ell)=\frac{1}{2} \mathrm{e}^{\lambda}\left(\widetilde{g}_{x-1}(\ell)+\widetilde{g}_{x+1}(\ell)\right),-\ell+1 \leqslant x \leqslant \ell-1$. Solving this differential equation with boundary condition yields $\widetilde{g}_{x}(\ell)=\cos \left(c_{\lambda} x\right) / \cos \left(c_{\lambda} \ell\right)$, with $c_{\lambda}=\arccos (\exp (-\lambda))$, and, setting $x=0$, (55) follows.

Remark. - Similarly, we get $E_{x}\left[\widetilde{\tau}_{\ell}\right]=\ell^{2}-x^{2}$. In particular, $E_{0}\left[\widetilde{\tau}_{\ell_{1}}\right]=\mathbf{E}_{\omega}\left[\tau_{\ell_{1}}\right]=\ell_{1}^{2}$ and this implies

$$
\mathbf{E}\left[\tau_{\ell_{1}}\right]=\mathbf{E}\left[\ell_{1}^{2}\right] .
$$

We will also use the following exit time asymptotics for simple random walk.

LEMMA 5. - In the setting of Lemma 4 , for $\ell=\ell(n)$ increasing with $\ell(n)^{2} / n \rightarrow 0$ and $c>0$, we have

$$
\lim _{n \rightarrow \infty} \frac{\ell(n)^{2}}{n} \log P_{0}\left[\bar{\tau}_{\ell(n)} \geqslant c n\right]=-c \frac{\pi^{2}}{8} .
$$

The proof of Lemma 5 is standard, using the explicit formula in [14], p. 243.

Proof of (24). - To show the lower bound, one proceeds as in the proof of (17), picking the largest interval without nodes. More precisely, let $m_{n}:=\inf \left\{j: \ell_{j}=\ell_{\max }(n)\right\}$ and $\delta>0$. We have

$$
\mathbf{P}_{\omega}\left[T_{n} \geqslant n u\right] \geqslant \mathbf{P}_{\omega}\left[\tau_{m_{n}} \geqslant n\left(u-\frac{1}{v_{\alpha}}+\delta\right)\right] \cdot \mathbf{P}_{\omega}\left[\sum_{1 \leqslant j \leqslant N_{\ell}(n), j \neq m_{n}} \tau_{\ell_{j}} \geqslant n\left(\frac{1}{v_{\alpha}}-\delta\right)\right] .
$$

The second term on the r.h.s. of (58) goes to 1, since due to (31) and (32),

$$
\frac{1}{n} \sum_{j=1}^{N_{\ell}(n)} \tau_{\ell_{j}} \underset{n \rightarrow \infty}{\longrightarrow} \frac{\mathbf{E}\left[\tau_{\ell_{1}}\right]}{\mathbf{E}\left[\ell_{1}\right]}=\frac{1}{v_{\alpha}} \quad \text { P-a.s. }
$$

For the first term on the r.h.s. of (58), (57) yields

$$
\frac{\ell_{\max }(n)^{2}}{n} \log \mathbf{P}_{\omega}\left[\tau_{m_{n}} \geqslant n\left(u-\frac{1}{v_{\alpha}}+\delta\right)\right] \underset{n \rightarrow \infty}{\longrightarrow}-\frac{\pi^{2}}{8}\left(u-\frac{1}{v_{\alpha}}+\delta\right) \quad \text { P-a.s. }
$$


and since $\lim _{n \rightarrow \infty} \ell_{\max }(n) / a_{n}=1$, P-a.s., the lower bound in (24) follows by letting $\delta \rightarrow 0$. To show the upper bound in (24), let $\delta \in] 0,1[$ and

$$
\lambda_{n}:=-\log \left(\cos \frac{\pi(1-\delta)}{2\left(a_{n}+d_{n}\right)}\right) .
$$

Due to (55), we have

$$
\mathbf{P}_{\omega}\left[T_{n} \geqslant n u\right] \leqslant \mathbf{E}_{\omega}\left[\mathrm{e}^{\lambda_{n} T_{n}}\right] \cdot \mathrm{e}^{-\lambda_{n} n u}=\prod_{j=1}^{N(n)} \frac{1}{\cos \left(\ell_{j} \arccos \mathrm{e}^{-\lambda_{n}}\right)} \cdot \mathrm{e}^{-\lambda_{n} n u} \quad \mathbf{P} \text {-a.s. }
$$

and, for P-a.a. $\omega$, due to (54) and (23), the r.h.s. is well-defined for $n$ large enough. Taking logarithms and multiplying with $a_{n}^{2} / n$ yields

$$
\frac{a_{n}^{2}}{n} \log \mathbf{P}_{\omega}\left[T_{n} \geqslant n u\right] \leqslant \frac{a_{n}^{2}}{n} \sum_{j=1}^{N(n)} \log \frac{1}{\cos \left(\ell_{j} \frac{\pi(1-\delta)}{2\left(a_{n}+d_{n}\right)}\right)}-a_{n}^{2} \lambda_{n} u \quad \text { P-a.s. }
$$

We check that, w.l.o.g., (23) still holds true if we replace $\ell_{\max }(n)$ with $\left.\ell_{\operatorname{MAX}}(n)\right)$, and conclude that

$$
d(\delta):=\cos \frac{\pi(1-\delta) \ell_{\mathrm{MAX}}(n)}{2\left(a_{n}+d_{n}\right)}>0 \quad \text { P-a.s. }
$$

for $n$ large enough, and the first term on the r.h.s of (62) is well-defined. Note that since $z^{-2} \log (\cos z) \rightarrow-1 / 2$ for $z \rightarrow 0$, we have for the second term on the r.h.s of (62), plugging in the value of $\lambda_{n}$,

$$
a_{n}^{2} \lambda_{n} u=-a_{n}^{2} u \log \left(\cos \frac{\pi(1-\delta)}{2\left(a_{n}+d_{n}\right)}\right) \underset{n \rightarrow \infty}{\longrightarrow} \frac{\pi^{2}}{8} u(1-\delta)^{2} .
$$

Let $\gamma>2$ be as in Theorem 2. For each $d>0$, there is $c=c(d)$ such that $-\log x \leqslant$ $1-x+c(1-x)^{\gamma / 2}$ for $d \leqslant x \leqslant 1$. Since $\cos x \geqslant 1-x^{2} / 2$, we also have

$$
-\log (\cos x) \leqslant \frac{x^{2}}{2}+c\left(\frac{x^{2}}{2}\right)^{\gamma / 2}
$$

for $d \leqslant x \leqslant 1$. Therefore, for $c=c(\delta)$ large enough,

$$
\frac{a_{n}^{2}}{n} \sum_{j=1}^{N(n)} \log \frac{1}{\cos \left(\ell_{j} \frac{\pi(1-\delta)}{2\left(a_{n}+d_{n}\right)}\right)} \leqslant \frac{a_{n}^{2}}{n} \sum_{j=1}^{N(n)} \ell_{j}^{2} \frac{\pi^{2}(1-\delta)^{2}}{8\left(a_{n}+d_{n}\right)^{2}}+c \frac{a_{n}^{2}}{n} \sum_{j=1}^{N(n)} \ell_{j}^{\gamma} \frac{\pi^{\gamma}(1-\delta)^{\gamma}}{8^{\gamma / 2}\left(a_{n}+d_{n}\right)^{\gamma}} .
$$

For the first term on the r.h.s of (64), note that

$$
\begin{aligned}
\lim _{n \rightarrow \infty} \frac{a_{n}^{2}}{n} \sum_{j=1}^{N(n)} \ell_{j}^{2} \frac{\pi^{2}(1-\delta)^{2}}{8\left(a_{n}+d_{n}\right)^{2}} & =\frac{\pi^{2}(1-\delta)^{2}}{8} \lim _{n \rightarrow \infty} \frac{a_{n}^{2}}{\left(a_{n}+d_{n}\right)^{2}} \frac{N(n)}{n} \frac{1}{N(n)} \sum_{j=1}^{N(n)} \ell_{j}^{2} \\
& =\frac{\pi^{2}(1-\delta)^{2}}{8} \frac{1}{\mathbf{E}\left[\ell_{1}\right]} \mathbf{E}\left[\ell_{1}^{2}\right]=\frac{\pi^{2}(1-\delta)^{2}}{8} \frac{1}{v_{\alpha}} \quad \text { P-a.s. }
\end{aligned}
$$


where we used (31), the ergodic theorem and the convergence of $d_{n} / a_{n}$ to 0 in the middle inequality and (10) in the last inequality. The same argument, using the assumption $\mathbf{E}\left[\ell_{1}^{\gamma}\right]<\infty$, shows that the second term on the r.h.s of (64) goes to 0 . We proved

$$
\limsup _{n \rightarrow \infty} \frac{a_{n}^{2}}{n} \log \mathbf{P}_{\omega}\left[T_{n} \geqslant n u\right] \leqslant(1-\delta)^{2} \frac{\pi^{2}}{8}\left(\frac{1}{v_{\alpha}}-u\right) \quad \text { P-a.s. }
$$

and since $\delta \in] 0,1[$ was arbitrary, the upper bound in (24) follows by letting $\delta \rightarrow 0$.

Proof of Theorem 3. - Lower bound: Let $h_{n}$ be an increasing function. We have

$$
\mathbf{P}\left[T_{n} \geqslant n u\right] \geqslant \mathbf{P}\left[\ell_{\max }(n) \geqslant h_{n}\right] P_{1}\left[\bar{\tau}_{h_{n}} \geqslant n u\right] .
$$

For the second term on the r.h.s. of (67), we have, using (39),

$$
P_{1}\left[\bar{\tau}_{h_{n}} \geqslant n u\right] \geqslant\left(1-\frac{1}{\rho^{h_{n}-1}}\right)^{n u} .
$$

Hence, choosing $h_{n}=\log n / \log \rho$,

$$
\frac{1}{\varphi(n)} \log \mathbf{P}\left[T_{n} \geqslant n u\right] \geqslant \frac{1}{\varphi(n)} \log \mathbf{P}\left[\ell_{\max }(n) \geqslant \frac{\log n}{\log \rho}\right]+\frac{1}{\varphi(n)} n u \log \left(1-\frac{\rho}{n}\right)
$$

and, using (26), (69) implies that

$$
\liminf _{n \rightarrow \infty} \frac{1}{\varphi(n)} \log \mathbf{P}\left[T_{n} \geqslant n u\right] \geqslant-\kappa\left(\frac{1}{\log \rho}\right) .
$$

Upper bound: Let $\varepsilon>0$ and $h_{n}=h_{n}(\varepsilon)=(1-\varepsilon) \log n / \log \rho$. We have

$$
\mathbf{P}\left[T_{n} \geqslant n u\right] \leqslant \mathbf{P}\left[\ell_{\operatorname{MAX}}(n) \geqslant h_{n}\right]+\mathbf{P}\left[T_{n} \geqslant n u, \ell_{\mathrm{MAX}}(n)<h_{n}\right] .
$$

We will show that

$$
\frac{1}{\varphi(n)} \log \mathbf{P}\left[T_{n} \geqslant n u, \ell_{\mathrm{MAX}}(n)<h_{n}\right] \underset{n \rightarrow \infty}{\longrightarrow}-\infty .
$$

We check that (26) still holds true if we replace $\ell_{\max }(n)$ with $\ell_{\operatorname{MAX}}(n)$. Then, using (26), we see from (71) and (72) and the continuity of $\kappa(\cdot)$ that

$$
\begin{aligned}
\limsup _{n \rightarrow \infty} \frac{1}{\varphi(n)} \log \mathbf{P}\left[T_{n} \geqslant n u\right] & \leqslant \lim _{\varepsilon \rightarrow 0} \lim _{n \rightarrow \infty} \frac{1}{\varphi(n)} \log \mathbf{P}\left[\ell_{\operatorname{MAX}}(n) \geqslant h_{n}(\varepsilon)\right] \\
& \leqslant-\kappa\left(\frac{1}{\log \rho}\right) .
\end{aligned}
$$


To show (72), let $\delta>0$ and $M_{\delta}:=(1+\delta) / \mathbf{E}\left[\ell_{1}\right]$ and note that

$$
\mathbf{P}\left[T_{n} \geqslant n u, \ell_{\operatorname{MAX}}(n)<h_{n}\right] \leqslant \mathbf{P}\left[N(n) \geqslant\left[n M_{\delta}\right]\right]+\mathbf{P}\left[\sum_{j=1}^{\left[n M_{\delta}\right]} \bar{\tau}_{\ell_{j, n}} \geqslant n u\right],
$$

where $\ell_{j, n}, j \in \mathbb{N}$, are i.i.d. with

$$
\mathbf{P}\left[\ell_{j, n}=k\right]=\frac{\mathbf{P}\left[\ell_{1}=k\right]}{\mathbf{P}\left[\ell_{1} \leqslant\left[h_{n}\right]\right]} \quad \text { for } k=1,2, \ldots,\left[h_{n}\right]
$$

and $\bar{\tau}_{\ell_{j, n}}, j \in \mathbb{N}$, are i.i.d. The distribution of $\bar{\tau}_{\ell_{j, n}}$, given $\ell_{j, n}$, is defined as in Lemma 2 . For the first term on the r.h.s of (74), we have

$$
\mathbf{P}\left[N(n) \geqslant\left[n M_{\delta}\right]\right]=\mathbf{P}\left[\sum_{j=1}^{\left[n M_{\delta}\right]-1} \ell_{j}<n\right] \leqslant \mathbf{P}\left[\frac{1}{\left[n M_{\delta}\right]} \sum_{j=1}^{\left[n M_{\delta}\right]-1} \ell_{j}<\frac{n}{\left[n M_{\delta}\right]}\right]
$$

Since $\ell_{j}$ are i.i.d., nonnegative random variables with expectations strictly larger than $1 / M_{\delta}$, the last term is decaying exponentially in $n$. In particular, since $\varphi(n) / n \rightarrow 0$, we have

$$
\frac{1}{\varphi(n)} \log \mathbf{P}\left[N(n) \geqslant\left[n M_{\delta}\right]\right] \underset{n \rightarrow \infty}{\longrightarrow}-\infty .
$$

Let $C>0$. We can estimate the second term on the r.h.s. of (74) as follows:

$$
\mathbf{P}\left[\sum_{j=1}^{\left[n M_{\delta}\right]} \bar{\tau}_{\ell_{j, n}} \geqslant n u\right] \leqslant \mathbf{E}\left[\exp \left(\frac{C \varphi(n)}{n} \bar{\tau}_{\ell_{1, n}}\right)\right]^{\left[n M_{\delta}\right]} \exp (-C \varphi(n) u) .
$$

We will show that

$$
\limsup _{n \rightarrow \infty} \frac{n}{\varphi(n)} \log \mathbf{E}\left[\exp \left(\frac{C \varphi(n)}{n} \bar{\tau}_{\ell_{1, n}}\right)\right] \leqslant C \mathbf{E}\left[\tau_{\ell_{1}}\right] .
$$

Together with (74)-(76), this implies that

$$
\limsup _{n \rightarrow \infty} \frac{1}{\varphi(n)} \log \mathbf{P}\left[T_{n} \geqslant n u, \ell_{\mathrm{MAX}}(n)<h_{n}\right] \leqslant C\left(\mathbf{E}\left[\tau_{\ell_{1}}\right] M_{\delta}-u\right) .
$$

But, recalling (6), for $u>1 / v_{\alpha}$ we can choose $\delta$ small enough such that the last term is negative, and since $C>0$ was arbitrary, this proves (72). It remains to prove (77). Using the inequalities $\log x \leqslant x-1$ and $\mathrm{e}^{x}-1 \leqslant x+x^{2} \mathrm{e}^{x}$, we have

$$
\begin{aligned}
& \frac{n}{\varphi(n)} \log \mathbf{E}\left[\exp \left(\frac{C \varphi(n)}{n} \bar{\tau}_{\ell_{1, n}}\right)\right] \\
& \quad \leqslant C \mathbf{E}\left[\bar{\tau}_{\ell_{1, n}}\right]+C^{2} \frac{\varphi(n)}{n} \mathbf{E}\left[\left(\bar{\tau}_{\ell_{1, n}}\right)^{2} \exp \left(\frac{C \varphi(n)}{n} \bar{\tau}_{\ell_{1, n}}\right)\right]
\end{aligned}
$$




$$
\leqslant C \mathbf{E}\left[\bar{\tau}_{\ell_{1}}\right]+C^{2} \frac{\varphi(n)}{n} \mathbf{E}\left[\left(\bar{\tau}_{\ell_{1, n}}\right)^{3}\right]^{2 / 3} \mathbf{E}\left[\exp \left(\frac{3 C \varphi(n)}{n} \bar{\tau}_{\ell_{1, n}}\right)\right]^{1 / 3} .
$$

Due to (46) and our assumption $\mathbf{E}\left[\rho^{3 \ell_{1}}\right]<\infty$, we have

$$
\limsup _{n \rightarrow \infty} \mathbf{E}\left[\left(\bar{\tau}_{\ell_{1, n}}\right)^{3}\right] \leqslant \mathbf{E}\left[\left(\tau_{\ell_{1}}\right)^{3}\right]<\infty
$$

Since $\ell_{1, n} \leqslant\left[h_{n}\right]$, we see from (80) that it suffices to show that for each $C>0$,

$$
\limsup _{n \rightarrow \infty} \mathbf{E}\left[\exp \left(\frac{C \varphi(n)}{n} \bar{\tau}_{\left[h_{n}\right]}\right)\right]<\infty
$$

But now we can use formula (44), plugging in $\ell=\ell_{n}=\left[h_{n}\right]$ and $\lambda=\lambda_{n}=C \varphi(n) / n$. Analysing the terms in (44), we see that $\lambda_{n} \rightarrow 0, \eta_{2}\left(\lambda_{n}\right) \rightarrow 1$ and $\eta_{1}\left(\lambda_{n}\right) \rightarrow \rho$. We show, with the same reasoning as in the proof of (72) in [7], that

$$
\eta_{2}^{h_{n}} \underset{n \rightarrow \infty}{\longrightarrow} 1 \text { and } \eta_{1}^{h_{n}}\left(\mathrm{e}^{\lambda_{n}} \eta_{2}-1\right) \underset{n \rightarrow \infty}{\longrightarrow} 0
$$

and this proves (82). For the proof of (28) from (27), note that $\mathbf{P}\left[X_{n} / n \leqslant v\right]$ is comparable to $\mathbf{P}\left[T_{[n v]} \geqslant n\right]$ by renewal duality, as in Lemma 3 , and use the fact that $\varphi$ is slowly varying; we refer to [3] for a more precise argument.

\section{Examples}

Example 1. - Assume that $\alpha$ is a product measure, with $\alpha_{1}:=\alpha\left(\omega_{0}=1\right)$ and $\alpha\left(\omega_{0}=p\right)=1-\alpha_{1}$. Then $\ell_{1}, \ell_{2}, \ldots$ are i.i.d. and $\ell_{1}$ has a geometric distribution with $\mathbf{P}\left[\ell_{1}=k\right]=\alpha_{1}\left(1-\alpha_{1}\right)^{k-1}, k=1,2, \ldots$ In this case, (14), (15) and (23) are satisfied with

$$
\begin{aligned}
a_{n} & =\frac{\log n}{-\log \left(1-\alpha_{1}\right)}, & b_{n} & =\frac{\log _{2} n}{-\log \left(1-\alpha_{1}\right)}, \\
c_{n} & =\frac{\log _{3} n}{-\log \left(1-\alpha_{1}\right)}, & d_{n} & =\frac{2 \log _{2} n}{-\log \left(1-\alpha_{1}\right)},
\end{aligned}
$$

and we have $\rho^{a_{n}}=n^{1 / s}$ with $s:=-\log \left(1-\alpha_{1}\right) / \log \rho$. Here, $a_{n}$ is given by the ErdôsRenyi law for longest runs, and (14), (15) and (23) follow from Theorem 2 in [4]. Hence, if $p<\frac{1}{2}$, we have for $v<v_{\alpha}$,

$$
\begin{aligned}
& \liminf _{n \rightarrow \infty} \frac{1}{n^{1-1 / s}} \log \mathbf{P}_{\omega}\left[X_{n} \leqslant n v\right]=-\infty \quad \text { P-a.s., } \\
& \limsup _{n \rightarrow \infty} \frac{1}{n^{1-1 / s}} \log \mathbf{P}_{\omega}\left[X_{n} \leqslant n v\right]=0 \quad \text { P-a.s. }
\end{aligned}
$$

For the general RWRE in an i.i.d. environment with "mixed drifts and positive speed", with $s$ defined by

$$
\int\left(\frac{1-\omega_{0}}{\omega_{0}}\right)^{s} \mathrm{~d} \alpha=1
$$


(86) was proved and (85) was conjectured in [6]. If $p<1 / 2$ in our example, the hypotheses of Theorem 3 are satisfied with $\varphi(n)=\log n$ and $\kappa(t)=-1-t \log \left(1-\alpha_{1}\right)$, and we have

$$
\lim _{n \rightarrow \infty} \frac{1}{\log n} \log \mathbf{P}\left[X_{n} \leqslant n v\right]=-(s-1)
$$

where $s=-\log \left(1-\alpha_{1}\right) / \log \rho$ as above. (87) agrees with Theorem 1.1 in [5]. For $p=\frac{1}{2}$ and $\alpha_{1 / 2}:=\left(1-\alpha_{1}\right)$, Theorem 2 yields that for $v<v_{\alpha}$,

$$
\lim _{n \rightarrow \infty} \frac{(\log n)^{2}}{n} \log \mathbf{P}_{\omega}\left[X_{n} \leqslant n v\right]=-\frac{\pi^{2}}{8}\left(\log \alpha_{1 / 2}\right)^{2}\left(1-\frac{v}{v_{\alpha}}\right) \quad \text { P-a.s. }
$$

If $\ell_{1}, \ell_{2}, \ldots$ are i.i.d. and $\mathbf{E}\left[\ell_{1}^{2}\right]<\infty$, recall the integral criteria for the growth of maxima of i.i.d. random variables in [9] and [10] to find functions $a_{n}, b_{n}, c_{n}$ and $d_{n}$ such that (14), (15) and (23) and our assumptions on $a_{n}$ are satisfied.

Example 2. - Assume that $\ell_{1}, \ell_{2}, \ldots$ are i.i.d. and $\mathbf{P}\left[\ell_{1}=k\right]=c \exp \left(-b k^{\gamma}\right), k=$ $1,2, \ldots$, where $\gamma \geqslant 1, b>0$ and

$$
c=\left(\sum_{k=1}^{\infty} \exp \left(-b k^{\gamma}\right)\right)^{-1} .
$$

Then (14), (15) and (23) are satisfied with

$$
\begin{aligned}
a_{n} & =\left(\frac{1}{b} \log n\right)^{1 / \gamma}, & b_{n} & =\left(\frac{1}{b} \log _{2} n\right)^{1 / \gamma}, \\
c_{n} & =\left(\frac{1}{b} \log _{3} n\right)^{1 / \gamma}, & d_{n} & =\left(\frac{2}{b} \log _{2} n\right)^{1 / \gamma} .
\end{aligned}
$$

Note that $\gamma<1$ would violate our assumption $\mathbf{E}\left[\rho^{\ell_{1}}\right]<\infty$. Hence, for $p<\frac{1}{2}$, (19), (20) and (25) are satisfied with $a_{n}$ as above. If $\gamma>1$ and $p<\frac{1}{2}$, Theorem 3 applies with $\varphi(n)=(\log n)^{\gamma}$ and $\kappa(t)=b t^{\gamma}$, and we have for each $u>\frac{1}{v_{\alpha}}$,

$$
\lim _{n \rightarrow \infty} \frac{1}{(\log n)^{\gamma}} \log \mathbf{P}\left[T_{n} \geqslant n u\right]=-\frac{b}{(\log \rho)^{\gamma}}
$$

and, for $v<v_{\alpha}$,

$$
\lim _{n \rightarrow \infty} \frac{1}{(\log n)^{\gamma}} \log \mathbf{P}\left[X_{n} \leqslant n v\right]=-\frac{b}{(\log \rho)^{\gamma}} .
$$

\section{Acknowledgements}

I would like to thank Ofer Zeitouni and Agoston Pisztora for several discussions. I thank Pal Révész and Balinth Toth, the organizers of the workshop "Random walks" at the Erdős center in Budapest, where these discussions took place, for inviting me to this very enjoyable meeting. I am also indepted to Didier Piau for his helpful comments. 
Finally, thanks are due to the referee for reading carefully and pointing out several mistakes in the first version of this paper.

\section{REFERENCES}

[1] Alili S., Asymptotic behaviour for random walks in random environments, J. Appl. Prob. 36 (1999) 1-16.

[2] Alili S., Chassaing P., Homogenization or slow diffusion for random walks with reflecting barriers, Tech. Rept. No 27, Prépublications de l'institut Élie Cartan, URA CNRS no ${ }^{0}$ 750, Université de Nancy, 1993.

[3] Comets F., Gantert N., Zeitouni O., Quenched, annealed and functional large deviations for one dimensional random walk in random environment, Probab. Theory Relat. Fields 118 (2000) 65-114.

[4] Deheuvels P., On the Erdős-Rényi Theorem for random fields and sequences and its relationships with the theory of runs and spacings, Zeitschrift für Wahrscheinlichkeitstheorie verw. Gebiete 70 (1985) 91-115.

[5] Dembo A., Peres Y., Zeitouni O., Tail estimates for one-dimensional random walk in random environment, Commun. Math. Phys. 181 (1996) 667-683.

[6] Gantert N., Zeitouni O., Quenched sub-exponential tail estimates for one-dimensional random walk in random environment, Commun. Math. Phys. 194 (1998) 177-190.

[7] Gantert N., Zeitouni O., Large deviations for one-dimensional random walk in a random environment - a survey, in: Révész P., Tóth B. (Eds.), Random Walks, Bolyai Society Mathematical Studies, Vol. 8, 1999, pp. 127-165.

[8] Greven A., den Hollander F., Large deviations for a random walk in random environment, Ann. Probab. 22 (1994) 1381-1428.

[9] Klass M., The minimal growth rate of partial maxima, Ann. Probab. 12 (1984) 380-389.

[10] Klass M., The Robbins-Siegmund series criterion for partial maxima, Ann. Probab. 13 (1985) 1369-1370.

[11] Pisztora A., Povel T., Large deviation principle for random walk in a quenched environment in the low speed regime, Ann. Probab. 27 (1999) 1389-1413.

[12] Pisztora A., Povel T., Zeitouni O., Precise large deviations estimates for one dimensional random walk in random environment, Probab. Theory Relat. Fields 113 (1999) 191-219.

[13] Solomon F., Random walks in random environment, Ann. Probab. 3 (1975) 1-31.

[14] Spitzer F., Principles of Random Walk, Springer, Berlin, 1976. 